\title{
Prediction of miRNA binding sites in mRNA
}

\author{
Anatoly Ivashchenko, Anna Pyrkova, Raigul Niyazova*, Aigul Alybayeva, Kirill Baskakov \\ Computer Science Laboratory, Al-Farabi Kazakh National University, Almaty - 050038, Kazakhstan, Raigul Niyazova - Email: \\ raiguln@mail.ru, *Corresponding author
}

Recieved May 26, 2016; Revised July 4, 2016; Accepted July 4, 2016; Published July 26, 2016

\begin{abstract}
:
In the present article, a solution for the problem of gene scanning for the purpose of prediction of binding sites of miRNA with matrix RNA (mRNA) is proposed. A mathematical model for the optimal process of scanning of genes and miRNA sequences was developed. An algorithm for scanning of genes using miRNA with one gap in the miRNA sequence and maximum (as a percentage ratio) free energy was developed and analysed with regard to the coincidence of miRNA and particular gene sites on the basis of complementarity. The constructed algorithm for scanning of genes using miRNA was parallelised and fragmented on the computational cluster with the use of MPJ tools.
\end{abstract}

Keywords: parallelized algorithm, cluster computing platform, Java MPI, miRNA, mRNA.

\section{Background:}

Searching for miRNA binding sites is difficult [1]. It is necessary to take into account the many characteristics (e.g., binding energy, degree of complementarity of nucleotides in the interaction of miRNA with mRNA, presence of non-interacting nucleotides, and multiplicity of binding sites located using one or more nucleotides). Recently, the number of identified miRNAs has increased to over 3,000 [2]. The up-to-date database mirBase.org contains approximately about 2,700 miRNA sequences. The volume of calculations for finding miRNA binding sites has increased significantly, and therefore requires the development of computer technology to increase the speed of calculations. In this study, we developed a program for prediction of miRNA binding sites that allowed us to increase by dozens of fold the speed of detection of miRNA binding sites. This program uses new additional characteristics for the interaction of nucleotides in the miRNA binding sites to the mRNA. Scanning of a genome enables hundreds of possible targets to be revealed for therapy of various diseases. Such scanning is important because knowledge of the interacting genes will allow the roles of the protein to be defined and will enable identification of intracellular processes that are disrupted in the disease.

\section{Methodology: \\ Mathematical model of scanning genes}

Scanning of genes is a process of consecutive comparison of sites of a gene with miRNA with possibility of adding one gap in the miRNA in positions with the 3rd on $n$-2-th, where $n$ indicates the nucleotide number (length) of miRNA. Thus, there is an assessment of all possible comparisons at one site of mRNA with miRNA that is defined according to the free energy value of the compared sequences. The option that is closer (in a percentage ratio) with regard to the free energy for coincidence of miRNA and a gene site is considered to be the best on the basis of complementarity. The mathematical model of the problem of scanning of genes can be formulated as follows:

Let $\left\{u_{l}\right\}, l=\overline{1, N}$ be a set of nucleotide or amino-acid sequences of miRNA, $N$ be the amount of sequences of miRNA,

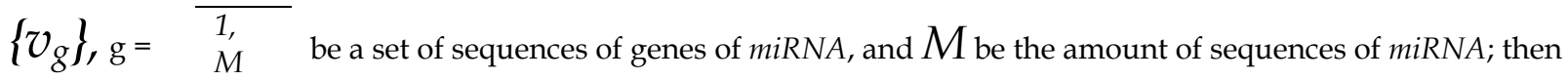

$\{\bar{u}, \bar{v}$ Number, Position, where, energy, score, length $\}$-scanning genes,

where Number is the order number, Position is a position of $\left\{\mathcal{U}_{l}\right\}$, in $\left\{\mathcal{V}_{g}\right\}$, where is an element from a set $\left\{5^{\prime} U T R, C D S\right.$, $\left.3^{\prime} U T R\right\}$ defining site arrangement area $\mathcal{u}_{l}$ in $v_{g}$, Energy is the value of free energy on the basis of a complementarity, Score is the value of $\Delta \mathrm{G} / \Delta \mathrm{Gm}$, and Length is the length of $\mathcal{U}_{l}$. 


\section{BIOINFORMATION \\ Discovery at the interface of physical and biological sciences}
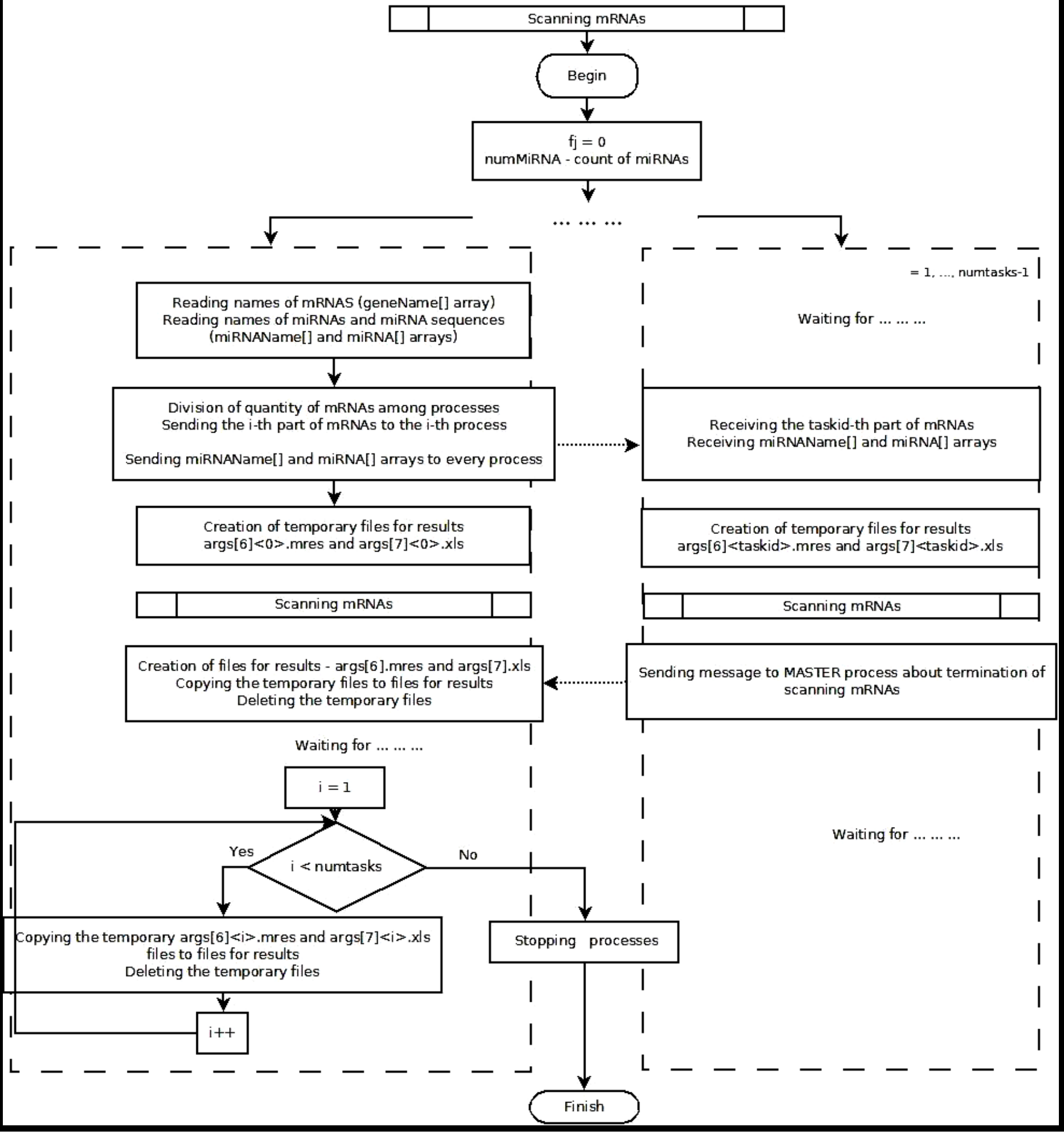

Figure 1: Flowchart of the scanning genes algorithm. 
Table 1: Comparative analysis of the linear, parallelized, and fragmented algorithms for scanning genes (time is specified in seconds)

Nodes Duration of processing (Average length $=21$ )

\begin{tabular}{lrrrr}
\hline Number of miRNA & 100 & 1000 & 10000 \\
\hline Linear algorithm & 3 & 54 & 532 \\
The parallelized algorithm & 15 & 1 & 3 & 8 \\
The fragmented algorithm & 15 & 1 & 7 & 53 \\
\hline
\end{tabular}

\begin{tabular}{|c|c|c|c|c|c|c|c|c|c|c|c|c|c|}
\hline & A & $\mathrm{B}$ & C & $\bar{D}$ & $\mathrm{~F}$ & G & $\mathrm{H}$ & 1 & $\bar{J}$ & K & L & $M$ & $N$ \\
\hline 1 & Target gene & $\underline{\text { miRNA }}$ & Position & nRNA domain & Energy & Score & Length & Binding site & mRNA fragment & t Oligopeptide & ORF1 & ORF2 & ORF3 \\
\hline 2 & TBC1D10B & miR-762(RP & \multicolumn{2}{|c|}{228 CDS } & -136 & 100 & 22 & geuecgged & ucggecgagac & \multicolumn{2}{|c|}{ SAETSAPAP APAPAPA } & LRPRPQP & SGPGPSP \\
\hline 3 & $B S N$ & miR-762(RP' & 184 & $\cos$ & -132 & 96,87 & 22 & geceggged & ggeggegecgg & \multicolumn{2}{|c|}{ GGAGPGPGPC ARAPAPA } & PGPRPRP & PGPGPGP \\
\hline 4 & CDKN1C & miR-762(RP' & 876 & $\mathrm{CDS}$ & -132 & 96,87 & 22 & gececgged & geggececgge & \multicolumn{2}{|c|}{ AMPAPAPAP I APAPAPA } & PRPRPRP & PGPGPGP \\
\hline 5 & CDKN1C & miR-762(RP & 882 & $\operatorname{cDS}$ & -132 & 96,87 & 22 & gececgged & ceggececage & \multicolumn{2}{|c|}{ PAPAPAP AP I $\triangle P A P A P A$} & PRPRPRP & PGPGPGP \\
\hline 6 & STK39 & miR-762(RP & 283 & $\operatorname{CDS}$ & -132 & 96,87 & 22 & gececgged & cecggeageuc & \multicolumn{2}{|c|}{ PGSSRGPGPC AP AP APA } & PRPRPRP & PGPGPGP \\
\hline 7 & TAF4 & miR-762(RP & 524 & $\operatorname{cDS}$ & -132 & 96,87 & 22 & geceggeed & gecggececgg & \multicolumn{2}{|c|}{ AGPGPGPGPC ARPRPRP } & PGPGPGP & PAPAPA \\
\hline 8 & CDKN1C & miR-762(RP & 858 & $\operatorname{CDS}$ & -129 & 95,31 & 22 & guegegged & ecggececgge & \multicolumn{2}{|c|}{ PAPAPVALPIVAAPAPA } & SRPRPQP & RGPGPSP \\
\hline 9 & CDKN1C & miR-762(RP & 888 & $\operatorname{cDS}$ & -129 & 95,31 & 22 & gececgged & ceagececgge & \multicolumn{2}{|c|}{ PAPAPAPAPI APAPAPA } & PRPRPPP & PGPGPRP \\
\hline 10 & RELA & miR-762(RP' & 1343 & $\operatorname{cDS}$ & -129 & 95,31 & 22 & geueugged & cuccagccaug & \multicolumn{2}{|c|}{ LQP WYQL WPF ÄL AQAP A } & L TIPRPQP & SGPGPSP \\
\hline 11 & SEMA3G & miR-762(RP & 61 & CDS & -129 & 95,31 & 22 & geueugged & cugeuccaugg & \multicolumn{2}{|c|}{ LLHGGSSGPS ALLAPAPA } & L WPQPRP & SGPSPGP \\
\hline 12 & STARD8 & miR-762(RP & 1657 & $\operatorname{cDS}$ & -129 & 95,31 & 22 & geugagged & aguggeacagg & \multicolumn{2}{|c|}{ SGTGRGOGPC AE AP APA } & LRPRPQP & OGPGPSP \\
\hline 13 & TBC1D10B & miR-762(RP & 240 & $\operatorname{cDS}$ & -129 & 95,31 & 22 & gececaged & ueugeuecgge & \multicolumn{2}{|c|}{ SAPAPAPAPI APAPAPA } & PQPRPQP & PSPGPSP \\
\hline 14 & UBE2O & miR-762(RP & 107 & $\operatorname{CDS}$ & -129 & 95,31 & 22 & geceagged & cegcageucec & \multicolumn{2}{|c|}{ PQLPLQPRPF AQAPAPA } & PRPRLQP & PGPGSSP \\
\hline 15 & PRRC2C & miR-762(RP & 5990 & $\operatorname{cDS}$ & -127 & 93,75 & 22 & geuucaged & cacuugececu & \multicolumn{2}{|c|}{ HLPLPQLQPC ASAPAP A } & LQPQPQP & FSPSPSP \\
\hline \multicolumn{14}{|c|}{$\begin{array}{llllllllll}\cdots & \cdots & \cdots & \cdots & \cdots & \cdots & \cdots & \cdots & \cdots\end{array}$} \\
\hline 94 & SH3BP1 & miR-762(RP & 1644 & $\operatorname{cDS}$ & -123 & 90,62 & 22 & geuecggel & ccagccaccac & \multicolumn{2}{|c|}{ PATTPAPAP I APAPAPA } & LRLRLQL & SGSGSSS \\
\hline 95 & SHANK3 & miR-762(RP & 3715 & $\cos$ & -123 & 90,62 & 22 & geceageed & cuguggcagag & \multicolumn{2}{|c|}{$L W Q S P C P A P C A Q P P G P A$} & PSPRAQP & PAPGPSP \\
\hline 96 & SMARCA4 & miR-762(RP' & 1051 & $\operatorname{cDS}$ & -123 & 90,62 & 22 & geceugged & uccgcaacagg & \multicolumn{2}{|c|}{ SATGPGPGPC ALALALA } & P WP WP WP & PGPGPGP \\
\hline 97 & STK39 & miR-762(RP & 278 & CDS & -123 & 90,62 & 22 & ecgeggece & gecggececgg & \multicolumn{2}{|c|}{ AGPGSSRGPCPRPRPRP } & RGPGPGP & AMPAPA \\
\hline 98 & SULT2B1 & miR-762(RP & 1170 & $\operatorname{CDS}$ & -123 & 90,62 & 22 & acuecaged & gugageccaga & \multicolumn{2}{|c|}{ VSPDPTP AP I TP AP APA } & LQPQPQP & SSPSPSP \\
\hline 99 & TAF4 & miR-762(RP & 505 & $\operatorname{cDS}$ & -123 & 90,62 & 22 & gegecgged & gecgegeugge & \multicolumn{2}{|c|}{ AALAARAGPCAPAPAPG } & RRPRPRA & AGPGPGP \\
\hline 100 & TAF4 & miR-762(RP & 535 & CDS & -123 & 90,62 & 22 & gececgged & ecegggecegg & \multicolumn{2}{|c|}{ PGPGPGPGPC AP APALA } & PRPRPUP & PGPGPGP \\
\hline 101 & TBC1D10B & miR-762(RP & 229 & $\operatorname{cDS}$ & -123 & 90,62 & 22 & cuecggeed & ucggecgagac & \multicolumn{2}{|c|}{ SAETSAPAPI LRPRPQP } & SGPGPSP & PAPAPA \\
\hline 102 & TBC1D10B & miR-762(RP' & 241 & CDS & -123 & 90,62 & 22 & ecceagece & ueugeuecgge & \multicolumn{2}{|c|}{ SAPAPAPAP I PQPRPQP } & PSPGPSP & PAPAPA \\
\hline 103 & TNFRSF21 & miR-762(RP' & 1988 & $\operatorname{CDS}$ & -123 & 90,62 & 22 & gecegaged & gagceceagce & \multicolumn{2}{|c|}{ EPQP AOPEPF ARAPSPA } & PEPHPQP & PSPIPSP \\
\hline 104 & TRIM47 & miR-762(RP & 298 & $\operatorname{cDS}$ & -123 & 90,62 & 22 & geueggged & cugcageuceg & LQLRQGSGPC & ARAPGP A & LGPRVRP & SGPGSGP \\
\hline 105 & TRIM65 & miR-762(RP & 310 & $\cos$ & -123 & 90,62 & 22 & gececgaur & cecgeceggga & PARDPGPDPC & APIPAPA & PRSRPRP & PDPGPGP \\
\hline 106 & USP51 & miR-762(RP & 460 & $\operatorname{cDS}$ & -123 & 90,62 & 22 & geceggeed & cecacececge & PTPASSPAP & ARPPPPP & PGPRPRR & PAPAPAD \\
\hline 107 & YAE1D1 & miR-762(RP & 481 & $\operatorname{cDS}$ & -123 & 90,62 & 22 & geceggeus & cacucecegeu & HSPLPRP APC & ARLRAPG & PGSGPQA & PAPGPRP \\
\hline 108 & $\mathrm{ZC} 3 \mathrm{H} 18$ & miR-762(RP & 2429 & CDS & -123 & 90,62 & 22 & ucueggues & ceguguecuca & PCPQPALGP & SRSPAPA & LGPRPQP & SVPGPSP \\
\hline
\end{tabular}

Figure 2: Results of processing the genome and miR-762.

For alignment of the nucleotide sequences of miRNA with mRNA, we assume the existence of only one admission on miRNA (lack of complementary couple for the hydrogen bond) that allows us to consider binding sites of mRNA longer than miRNA by one nucleotide. In this case, the regular structure of the spiral is broken, and a bulge exists. The free energy of binding of miRNA with mRNA for such a structure is less than that in an alternative case. The program determines the free energy of hybridisation $(\Delta \mathrm{G}, 100 \mathrm{~kJ} / \mathrm{mole})$ of miRNA with mRNA and the scheme of their interactions; it also calculates the relationship $\Delta \mathrm{G} / \Delta \mathrm{Gm}$, the levels of reliability (p), and the mRNA areas where the site (5'UTR, CDS or $\left.3^{\prime} \mathrm{UTR}\right)$ starting at the first nucleotide of the $5^{\prime} \mathrm{UTR}$ is located. $\Delta \mathrm{Gm}$ is equal to the free energy of binding of miRNA with a site in nucleotide sequence of miRNA completely complementary to it. The level of reliability (p) was defined on the basis of the value of $\Delta G$ and its standard deviation. The program outputs the scheme of the interaction of miRNA with mRNA; a site position in 5'UTR, CDS, or 3'UTR; and the free energy of interaction of miRNA with mRNA and its relative value from the maximum energy of binding of miRNA. In the program, the threshold value of this relationship is set, and this value prevents consideration of sites with weak free energy of binding. In this article, an algorithm for scanning of genes on the cluster is provided (Figure 1).

\section{Results and Discussion:}

The developed algorithm has advantages that are not present in known programs for prediction of binding sites of miRNA with mRNA. With using of the realized fragmented algorithm on the cluster platform www.ursa.kaznu.kz data for a human genome were processed about 2700 miRNAs. miR-762 has 108 sites in the CDS of mRNAs with $\Delta \mathrm{G} / \Delta \mathrm{Gm}$ values greater than or equal to 90\% (see Figure 2). miR-762 has 19 binding sites on the mRNA of the CDKN1C gene, 7 binding sites on the mRNA of the TAF4 gene, 6 binding sites on the mRNA of the BSN gene. The binding sites are highly conserved. Nucleotide sequences of the binding sites in CDS encode olygopeptides containing mainly alanine and proline depending on the reading frame (see Figure1). On a 
cluster 24 nodes were involved, the fragmented algorithm broke input data at first into 24 parts, then 9 of them into 24 fragments as 15 nodes finished the operation before remaining ones.

The algorithm of processing took 31,5 times less time, than when the sequential algorithm of scanning which processes the same data.

In the literature, there are many data regarding the value of the free energy of hydrogen bonds between nucleotides in water solution [3]. However, there is high variability in the value of the free energy of this bond and it is difficult to give preference to certain data [4], [5]. It is important to know the relative relationships of the free energy of hydrogen bonds between nucleotides, as they are necessary for the formation of secondary and tertiary structures in RNA. The analysis of the free energy of the hydrogen bond arising between nucleotides during intramolecular interactions of mRNA in the formation of its secondary structure showed that three bonds formed between the $G$ and $C$ nucleotides, two between $A$ and $U$, and one between $G$ and $U$ and between $A$ and $S$. The relationship of the free energy of the hydrogen bond in G-C and A-U pairs approximately corresponds to the relationship of the forces of their 3:2 interaction ( $0.188 \mathrm{nNewton}$ and $0.125 \mathrm{nNewton}$, respectively) [6]. The value of the free energy of one hydrogen bond between nucleotides varies in the range from -0.7 to $-1.6 \mathrm{kcal} / \mathrm{mol}$ [7]. In this algorithm, the free energy of the interaction of nucleotides resulting from hydrogen communication was considered as equal to $6.368 \mathrm{~kJ} / \mathrm{mol}$ and $4.246 \mathrm{~kJ} / \mathrm{mol}$ for G-C and A-U pairs, and $2.123 \mathrm{~kJ} / \mathrm{mol}$ for G-U and A-S pairs, respectively.

The distance between nucleotides in G-C and A-U pairs is 1.03 $\mathrm{nm}$, whereas that between nucleotides in the G-U pair is $1.02 \mathrm{~nm}$, and that between nucleotides in the A-S pair is $1.04 \mathrm{~nm}$ [8]. Therefore, formation of hydrogen bonds between these pairs of nucleotides enables the two-chained structure of mRNA to have a spiral form similar to that of DNA. Such a structure of mRNA without hydrogen bonds is stabilised by stacking interactions between the nitrogenous bases [9]. The distance between nucleotides in purine-purine and pyrimidine-pyrimidine pairs significantly differs from 1.03 nanometers; the distances for A-A, G-A, and G-G are equal to $1.23 \mathrm{~nm}, 1.25 \mathrm{~nm}$, and $1.25 \mathrm{~nm}$, respectively. In pyrimidine-pyrimidine pairs, the distances between nucleotides also significantly differed from 1.03 nanometers; the distances between nucleotides for $\mathrm{C}-\mathrm{C}, \mathrm{U}-\mathrm{U}$, and $\mathrm{U}-\mathrm{C}$ was equal to $0.85 \mathrm{~nm}, 0.81 \mathrm{~nm}$, and $1.18 \mathrm{~nm}$, respectively. Therefore, in such pairs, hydrogen bonds are not formed, and these pairs will disrupt the regular structure of two-chained miRNA using the mRNA-reducing ability of the RISC complex (RNA-induced silencing complex). Therefore, in the program, such couples of hydrogen bonds were not considered.

According to Table $\mathbf{1}$ it is obvious that the fragmented algorithm in comparison with the parallelized algorithm gives advantage on handling speed on large volumes of data. In case of increasing quantity of nodes of a supercomputer data are distributed on smaller fragments and this increases the general speed of execution of the program. So, for example, when using 15 nodes of a supercomputer the general speed of execution of the program, when handling 10000 miRNAs, becomes 6,6 times higher, than when handling the same number of data by the parallelized algorithm.

The technology of the fragmented programming allows not only distributing data and functions between nodes, and, besides, promotes optimum work of a supercomputer in general. So there is an opportunity to redistribute loading between nodes on the program course if any nodes have finished the work before other nodes, and other nodes are still loaded. While during the work with the parallelized algorithm such opportunity needs to be programmed separately.

\section{Conclusion:}

In this study, we have shown using of the realized fragmented algorithm on the cluster platform www.ursa.kaznu.kz data for seach binding sites of miR-762 in mRNAs of human genes. MiR762 has 108 sites in the CDS of mRNAs with $\Delta \mathrm{G} / \Delta \mathrm{Gm}$ values greater than or equal to $90 \%$. The feature of this miRNA is the presence of multiple binding sites in the mRNA of its target genes. miR-762 was high in both breast cancer cell lines and specimens, and its overexpression increased breast cancer cell proliferation and invasion [10]. miR-762 is involved in the matrix mineralization in mature osteoblasts, vascular smooth muscle cell calcification and other processes [11-13]. miR-762 is identified as a potential biomarker for monitoring the state of immunosuppression, allograft nerve [14]. Therfore study the characteristics of miR-762 binding sites is important for undestanding its functions.

\section{References:}

[1] Ivashchenko A. et al. Bioinformation. 201612 (1): 15 [PMID: 27212839]

[2] Eric Londina et al. Proc Natl Acad Sci USA. 2015 112: E1106 [PMID: 25713380]

[3] Guckian K. et al. J.Am.Chem.Soc. 1996 118: 8182 [PMID: ]

[4] Turner D. et al. J. Am. Chem. Soc. 1987 109: 3783

[5] Sugimoto N. et al. Biochemistry. (1987) 14: 4554

[6] Boland T. \& Ratner B. Proc. Natl. Acad. Sci. USA 199592 : 5297

[7] Kool E. Annu. Rev. Biophys. Biomol. Struct. 2001 30: 1

[8] Leontis N. et al. Nucleic Acids Res. 2002 30: 3497

[9] Richard A., et al. Biophysical Journal. 1995 69: 1528-1535

[10] Li Y. et al. Cell Prolif. 2015 48: 643

[11] Gao F. et al. Int J Biol Sci. 2015 11: 109

[12] Gui T. et al. Lab Invest. 2012 92: 1250

[13] Mo X. et al. Intractable Rare Dis Res.2014 3: 12

[14] Rau C. et al. J Biomed Sci. 2013 20: 64

Edited by $\mathbf{P}$ Kangueane

Citation: Ivashchenko et al. Bioinformation 12(4): 237-240 (2016) License statement: This is an Open Access article which permits unrestricted use, distribution, and reproduction in any medium, provided the original work is properly credited. This is distributed under the terms of the Creative Commons Attribution License. 